\title{
Assimilation and contrast of perceived length depend on temporal factors
}

\author{
KEVIN JORDAN \\ San Jose State University, San Jose, California \\ and \\ JOHN UHLARIK \\ Kansas State University, Manhattan, Kansas
}

\begin{abstract}
The parallel lines configuration produces assimilation of linear extent when the contextual and focal lines are presented simultaneously and contrast when the contextual line is presented prior to the focal judgment. The interpretation of the shift from assimilation to contrast is confounded by the covariation of mode of presentation (simultaneous or sequential) of contextual and focal stimuli and the duration of contextual exposure in previous research. This confound was removed by varying contextual exposure duration within the sequential mode of presentation (Experiment 1) and by combining the simultaneous and sequential modes of presentation (Experiment 2). The results indicated that the shift from assimilation to contrast is determined by the mode of presentation of contextual and focal stimuli. The data are discussed as they relate to Coren and Girgus's (1978) "pool and store" model of assimilation and contrast.
\end{abstract}

Visual contours often interact to produce systematic distortions in the apparent value of some parameter of form. These interactions can be observed in two manners. First, the contours can be presented simultaneously so that one stimulus (the contextual stimulus) produces a distortion of the stimulus being judged (the focal stimulus). This phenomenon is usually referred to as visual illusion. A second kind of interaction, called an aftereffect, occurs when the focal stimulus is perceptually distorted subsequent to inspection of a contextual stimulus. Both illusions and aftereffects can occur in two directions. When the perception of the focal stimulus is shifted away from the value of the contextual stimulus, the effect is termed "contrast," and when the perception of the focal stimulus is shifted toward the contextual stimulus, the effect is termed "assimilation."

Illusions and aftereffects occur in the same direction for many parameters of form. For example, both orientation illusion (Blakemore, Carpenter, \& Georgeson, 1970; Jastrow, 1982) and orientation aftereffect (the tilt aftereffect; Gibson \& Radner, 1937) are contrast effects. Also, spatial-frequency illusion (Klein, Stromeyer, \& Ganz, 1974) and spatial-frequency aftereffect (Blakemore, Nachmias, \& Sutton, 1970) are contrast effects. Distortions of linear extent represent an interesting exception to this general rule, because the directions of illusion and aftereffect are opposite; length illusion (e.g., the MüllerLyer illusion and the illusion of parallel lines) is an as-

The authors would like to thank Diane Schiano and two anonymous reviewers for their comments on earlier versions of the manuscript. Requests for reprints should be sent to Kevin Jordan, Department of Psychology, San Jose State University, San Jose, CA 95192. similation effect, whereas length aftereffect (the aftereffect of parallel lines; Brigell \& Uhlarik, 1979) is a contrast effect.

There are two differences between the procedures generally used to establish length illusions and aftereffects which could account for the effects' being opposite in direction. The most obvious procedural difference is that the contextual and focal stimuli are presented simultaneously in the illusion paradigm but sequentially in the aftereffect paradigm. However, another important difference is that the contextual stimulus is generally inspected for a longer period in the aftereffect paradigm. For example, to establish a length aftereffect, Brigell and Uhlarik (1979) had observers inspect a contextual line for $60 \mathrm{sec}$ prior to making a focal judgment. On the other hand, in the illusion paradigm, contextual and focal stimuli are inspected for the same amount of time (by definition) and usually for a much shorter duration. Thus, interpretation of the shift from length assimilation to contrast is confounded by covariation of mode of presentation of contextual and focal stimuli (simultaneous vs. sequential) and duration of exposure to the contextual stimulus (same or longer than focal exposure).

Different models of perceptual distortions make different predictions about which factor produces the shift from assimilation to contrast. Coren and Girgus's (1978) "pool and store" model proposes that assimilation results from the pooling of all information in the visual array and that contrast is produced by successive sampling of the visual array. The critical variable in producing assimilation or contrast "is simply whether the stimuli are close enough to be glimpsed simultaneously and hence averaged, or whether they are distant enough to require successive sam- 
pling of the array"' (Coren \& Girgus, 1978, p. 171). The analogy to the length illusion and aftereffect paradigms is straightforward. When the contextual and focal lines are presented simultaneously and in close spatial proximity, as in the illusion paradigm, the result is assimilation; when the contextual line is inspected prior to focal presentation, as in the aftereffect paradigm, length contrast results. According to this model, then, the mode of presentation of contextual and focal stimuli is more important than the duration of contextual exposure in determining the direction of length distortions. On the other hand, many models propose that contrast is the result of the fatigue of feature-specific neural units produced by prolonged exposure to a contextual stimulus (cf. Coltheart, 1971). For this type of model, it does not seem to matter whether the focal line is present prior to or during contextual exposure. Rather, the important factor in contrast seems to be the duration of contextual exposure. Presumably, the greater the duration of inspection, the greater the buildup of neural fatigue. Possibly, the assimilation observed in the length-illusion, or simultaneous-presentation, condition is simply the result of there not having been enough time for the hypothetical fatigue to become established. Thus, this approach proposes that in producing the shift from assimilation to contrast of linear extent, the duration of contextual exposure is more important than the mode of presentation.

The purpose of the present experiments was to examine length illusion and length aftereffect using the parallellines array in order to determine the basis for their disparity in direction. One approach to the problem is to remove the confound between mode of presentation and duration of exposure to the contextual stimulus by holding one of these factors constant and systematically varying the other factor. The first experiment was designed to vary time of exposure to the contextual stimulus within one mode of presentation (sequential) in order to assess the effects of contextual exposure duration per se. The second experiment allowed an assessment of contextual exposure duration independent of a particular mode of presentation. This was accomplished by combining the simultaneous and sequential modes of presentation within a single procedure; the contextual stimulus was present both prior to and during the focal judgment, and duration of prior contextual exposure was varied.

\section{EXPERIMENT 1}

Experiment 1 was designed to remove the confound of mode of presentation of contextual and focal stimuli and duration of contextual exposure in the illusion and aftereffect of parallel lines. This confound has prevented unambiguous interpretation of the shift from length assimilation to length contrast. Duration of contextual exposure was varied within the sequential mode of presentation (the aftereffect paradigm) in order to initially assess the effect of contextual exposure duration, per se, on focal judgments. The sequential mode of presentation was chosen because pilot work had indicated that long contextual exposures were not effectively attained within the simultaneous mode of presentation (the illusion condition). Specifically, when the contextual and focal lines were presented simultaneously for durations as long as $60 \mathrm{sec}$, observers indicated that they had made their focal judgment very early in the 60-sec interval and simply "held" their response until it was to be reported at the end of the interval.

The durations of contextual exposure chosen for the present experiment were 5 and $60 \mathrm{sec}$. Sixty seconds was chosen because this was the duration used by Brigell and Uhlarik (1979) in the initial study of the aftereffect of parallel lines. Five seconds was chosen as an approximation of the duration of contextual exposure in the simultaneous presentation mode. For example, in an experiment on the illusion of parallel lines, Jordan (1982) allowed observers up to $10 \mathrm{sec}$ to make a focal judgment, but in most cases the observers reported their judgments prior to the 10-sec limit, with an average of about $5 \mathrm{sec}$. Thus, observers were exposed simultaneously to the contextual and focal stimuli for about $5 \mathrm{sec}$. By comparison, in the present experiment, observers were first exposed to the contextual stimulus for $5 \mathrm{sec}$ and then to the focal stimulus. The essential difference between the two procedures was the mode of presentation. If significant length contrast results from $5 \mathrm{sec}$ of contextual exposure prior to the presentation of the focal stimulus, it would seem that mode of presentation of the contextual and focal stimuli, per se, would be the important factor in determining whether length assimilation or length contrast occurs.

Finally, the present experiment also included a condition in which contextual and focal stimuli were presented simultaneously. This is simply a length illusion condition, and it was included to facilitate comparison of simultaneous and sequential modes of presentation.

\section{Method}

\section{Observers}

Fifty-four students in an introductory psychology course participated in the experiment in order to earn course credit. Eighteen observers were assigned each to the illusion, the 5-sec aftereffect, and the $60-\mathrm{sec}$ aftereffect conditions. The observers were required to have at least $20 / 30$ visual acuity with or without correction. The observers were run in individual sessions.

\section{Stimuli and Design}

The stimulus lines were made with white tape oriented horizontally on a black background. The stimuli were then photographed using high-contrast Kodalith film that produced $35-\mathrm{mm}$ slide negatives (black lines on a white background), which were projected on the rear of a translucent screen by a Kodak random-access slide projector. Stimulus presentation was controlled by a projection tachistoscope.

The specific lengths of the focal and contextual lines were chosen to produce maximum over- and underestimation of focal length. Brigell and Uhlarik (1979) reported maximum underestimation of focal length when the ratio of the contextual length to the focal length (the "framing" ratio) was between 0.5:1.0 and 0.8:1.0 and maximum overestimation when the framing ratio was between 1.25:1.0 and 2.0:1.0. In the present study, the projected lengths of the con- 
textual lines were $1.4,1.9,4.2$, and $5.6 \mathrm{~cm}$ and the focal length was $2.8 \mathrm{~cm}$, resulting in framing ratios of $0.5,0.67,1.5$, and 2.0 , respectively. The thickness of the lines on the viewing screen was $0.7 \mathrm{~mm}$, and the viewing distance was $77 \mathrm{~cm}$.

In the illusion condition, the contextual line and the focal line were on the same slide. The $2.8-\mathrm{cm}$ focal line was centered on the viewing screen, and the contextual line was centered $1.2 \mathrm{~cm}$ below the focal line. Thus, there were four test slides in the illusion condition-one slide each of contextual length $1.4,1.9,4.2$, and $5.6 \mathrm{~cm}$, all of which contained the $2.8-\mathrm{cm}$ focal line. The adapting array used in the length-afterefect conditions contained a single contextual line located $1.2 \mathrm{~cm}$ below a fixation point in the center of the viewing screen. Again, the length of this contextual line was $1.4,1.9,4.2$, or $5.6 \mathrm{~cm}$, and the subsequently presented $2.8-\mathrm{cm}$ focal line was centered on the viewing screen. To summarize the design: there were three levels of presentation of contextual and focal stimuli (simultaneous, 5-sec sequential, and 60-sec sequential) and four levels of contextual length $(1.4,1.9,4.2$, and $5.6 \mathrm{~cm})$. The mode of presentation of contextual and focal stimuli was a between-subjects factor.

Focal judgments were obtained using a graded series comparison scale (cf. Coren \& Girgus, 1972) located $9.5 \mathrm{~cm}$ to the right of the midpoint of the focal line. The scale consisted of 11 horizontally oriented lines, labeled " $A$ " through " $K$," which ranged from 2.3 to $3.3 \mathrm{~cm}$ in $1-\mathrm{mm}$ increments. Therefore, the point of objective equality (POE) on this scale for all test stimuli was " $F$," or $2.8 \mathrm{~cm}$. The lines on the graded-series scale were separated vertically by $5 \mathrm{~mm}$. In order to encourage observers to use a wide range of the response scale, two filler stimuli with focal lengths of 2.4 and $3.2 \mathrm{~cm}$ were included in the design. Responses to these filler stimuli were not included in the data analysis.

To familiarize the observers with the use of the graded-series scale, there were also six practice stimuli, which consisted of only a focal line. The focal lengths for these stimuli were $2.4,2.6,2.8,2.8$, 3.0 , and $3.2 \mathrm{~cm}$.

\section{Procedure}

The observers viewed the projected image of the stimuli in an otherwise darkened room. The observers were familiarized with the range and the variety of the stimuli, as well as with the use of the graded series scale. Specifically, they were told that the task was to match the apparent length of the focal line to one of the 11 lines on the graded-series scale. If a focal line appeared to be intermediate in length to two lines on the scale, the observers were encouraged to interpolate using a 10 -point scale.

Illusion condition. The 18 observers in the illusion condition first proceeded through a random order of the six practice stimuli. These practice trials were followed by a random order of the four test and two filler stimuli. A trial consisted of the simultaneous presentation of the contextual and focal line for up to $10 \mathrm{sec}$, during which the focal judgment was to be made. There was a 10-sec dark interval separating each trial. After proceeding through the first random order of the test and filler stimuli, the observers proceeded through three more random orders of these six stimuli. In all, each of the 18 observers made 30 focal judgments-six practice judgments and four judgments of each of the four test and two filler stimuli. The entire procedure required approximately $10 \mathrm{~min}$ for each observer.

Aftereffect condition. The 18 observers in each of the aftereffect conditions also first proceeded through a random order of the six practice stimuli. The observers then proceeded through a random order of the four test and two practice trials. A trial consisted of either a 5- or 60-sec inspection of the contextual line, during which the observers were instructed to fixate the small spot in the center of the screen. This adaptation period was followed by the presentation of the focal line for up to $10 \mathrm{sec}$, during which the length judgment was to be made. The interstimulus interval was $1 \mathrm{sec}$ for both aftereffect conditions. Each trial was separated by a 20 -sec dark interval to allow any afterimages to dissipate. The observers then proceeded through three more random orders of the four test and two filler trials, with a 2-min break between the second and third random orders. In all, each of these 36 observers made 30 focal judgments-six practice judgments and four judgments of each of the four test and two filler stimuli. The procedure required approximately $18 \mathrm{~min}$ for each observer in the 5 -sec condition and $45 \mathrm{~min}$ for each observer in the 60 -sec condition.

\section{Results and Discussion}

The responses to the $2.8-\mathrm{cm}$ "no-context" focal line contained in the practice series were averaged to estimate the point of subjective equality (PSE) for the $2.8-\mathrm{cm}$ focal line for each group of observers. The mean of the 36 responses (two judgments from each of 18 observers) was $2.66 \mathrm{~cm}$ in the illusion condition, $2.63 \mathrm{~cm}$ in the $5-\mathrm{sec}$ aftereffect condition, and $2.62 \mathrm{~cm}$ for the $60-\mathrm{sec}$ aftereffect condition. This consistent underestimation of objective focal length was also reported by Brigell and Uhlarik (1979). Focal judgments were converted into deviations from PSE by subtracting the PSE from the mean judged length of each test stimulus for the illusion condition and the aftereffect conditions. These data are presented in Figure 1. Clearly, there was substantial assimilation of focal length in the illusion condition in that contextual lines longer than the $2.8-\mathrm{cm}$ focal line (i.e., 4.2 and $5.6 \mathrm{~cm}$ ) resulted in overestimation of focal length, and lines shorter than the focal line (i.e., 1.4 and $1.9 \mathrm{~cm}$ ) produced underestimation. Additionally, there was contrast of focal length in the aftereffect conditions; contextual lines shorter than the $2.8 \mathrm{-cm}$ focal line resulted in overestimation of focal length, and, at least for the 60 -

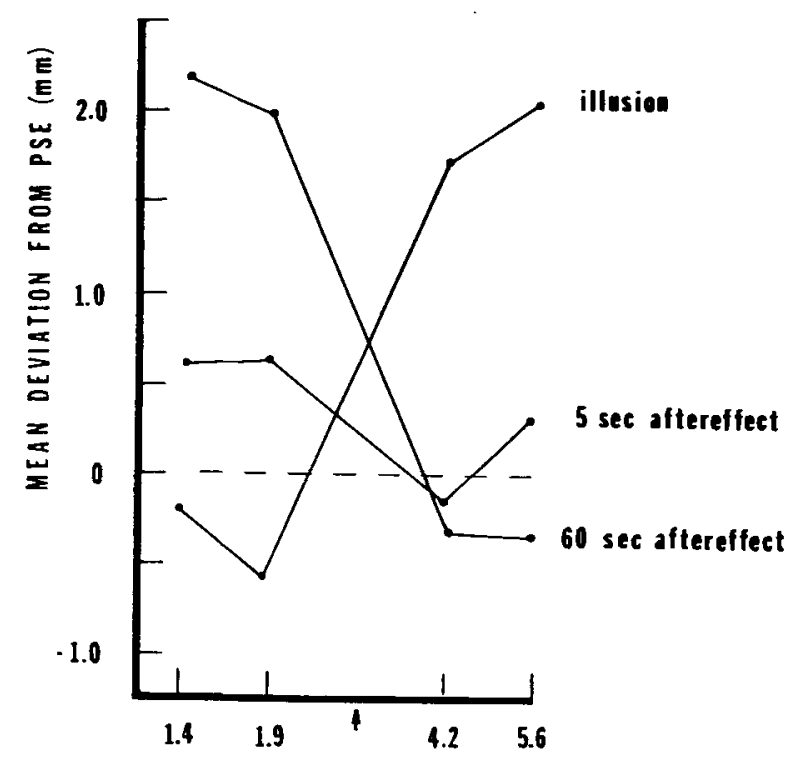

CONTEXTUAL LENGTH (cm)

Figure 1. Mean deviations from PSE in millimeters for the illusion, 5-sec aftereffect, and 60-sec aftereffect conditions of Experiment 1 . The illusion data indicate assimilation of perceived focal length; the aftereffect data indicate contrast. The arrow on the abscissa indicates the focal length $(2.8 \mathrm{~cm})$ for the test stimuli. 
sec condition, lines longer than the focal line produced underestimation.

The data for the 5- and 60-sec aftereffect conditions were analyzed separately in 4 (contextual lengths) $\times 4$ (replications) $\times 18$ (observers) within-subject analyses of variance. For the 5-sec condition, the main effect of contextual length was statistically significant $[F(3.51)=$ $8.84, p<.01]$, which indicates that the contextual lines affected focal judgments. The direction of this effect (see the curve labeled " $5 \mathrm{sec}$ " in Figure 1) indicates length contrast. Also, the effect of contextual length was significant in the 60-sec condition $[\mathrm{F}(3,51)=77.53, \mathrm{p}<.01]$, again indicating that the contextual lines affected focal judgments to produce length contrast. No other main effect or interactions reached statistical significance in either analysis.

Although the data indicate contrast of perceived focal length subsequent to both the 5- and the 60 -sec contextual exposures, the 5-sec aftereffect appears to be much less than the $60 \mathrm{sec}$ aftereffect. In order to test whether there was a difference in the magnitudes of the aftereffects, the data for the 5- and 60-sec conditions were analyzed in a 2 (exposure durations) $\times 4$ (contextual lengths) $\times 4$ (replications) $\times 18$ (observers) mixed analysis of variance. Exposure duration was a between-subjects factor. Again, the effect of contextual length was statistically significant $[\mathrm{F}(3,102)=74.41, \mathrm{p}<.01]$. The effect of exposure duration did not reach statistical significance $[F(1,34)=1.12]$. However, there was a significant exposure duration $\times$ contextual length interaction $[F(3,102)=28.98, p<.01]$, indicating that the slopes of the curves representing the 5- and 60-sec contrast effects (see Figure 1) were significantly different. The direction of the slope difference indicates that the contrast of perceived focal length resulting from $5 \mathrm{sec}$ of prior contextual exposure is significantly less than the contrast effect resulting from $60 \mathrm{sec}$ of prior contextual exposure.

In summary, the results of Experiment 1 indicate that mode of presentation, per se, is important in determining whether length assimilation or length contrast occurs. When the contextual and focal lines are examined simultaneously for about $5 \mathrm{sec}$, as in the illusion condition, length assimilation results; when the focal line is judged after $5 \mathrm{sec}$ contextual exposure, as in the 5 -sec aftereffect condition, length contrast results. Thus, when contextual exposure duration is held constant, variation of the mode of presentation of contextual and focal stimuli from simultaneous to sequential results in a shift from length assimilation to length contrast. However, the contrast produced in the 5-sec aftereffect condition appears to be substantially less than the assimilation produced by an equivalent duration of contextual exposure in the simultaneous (illusion) condition. Furthermore, within the sequential mode of presentation (the aftereffect conditions), variation of contextual exposure duration from 5 to $60 \mathrm{sec}$ results in a statistically significant increase in the magnitude of length contrast. Thus, it appears that both the difference in the mode of presentation and the contextual exposure duration are involved in the disparity in the distortions of perceived length produced by the length illusion and length aftereffect paradigms observed by Brigell and Uhlarik (1979).

Experiment 2 was designed to eliminate the differences in mode of presentation between the illusion and aftereffect paradigms by collapsing across them. Instead of presenting a contextual line either prior to or at the same time as the focal line, we combined the two procedures such that a contextual line was presented both prior to and during the focal judgment. Within this combined condition, contextual exposure duration was again varied in order to assess the effect of equal and unequal amounts of contextual and focal exposure on length distortion.

\section{EXPERIMENT 2}

Experiment 1 focused on the effect of contextual exposure duration within the aftereffect paradigm (i.e., sequential presentation of the contextual and focal stimuli). However, variation of contextual exposure duration was not systematically assessed within the illusion paradigm (i.e., simultaneous presentation). Experiment 2 used a procedure that allowed for the inclusion of the simultaneous presentation of the contextual and focal lines while varying prior contextual exposure in order to eliminate the methodological problems discussed in Experiment 1. This procedure involved combining the illusion and aftereffect paradigms by exposing an observer to the contextual line throughout a trial. Thus, an observer viewed a contextual line for a period of time $(5$ or $60 \mathrm{sec})$ and then was exposed to the focal line in the presence of that same contextual line (cf. Magnussen \& Kurtenbach's 1980, work on orientation illusion and aftereffect). This procedure has the effect of eliminating a distinction between the illusion and aftereffect paradigms and allows a direct assessment of contextual exposure duration independent of a particular mode of presentation.

\section{Observers \\ Method \\ Thirty-six students in an introductory psychology course partic- ipated in the experiment in order to earn course credit. The ob- servers were required to have at least $20 / 30$ visual acuity with or without correction. The observers were run in individual sessions.}

\section{Stimuli and Design}

The stimuli were constructed and photographed in the same manner as in the previous experiment. The contextual stimulus presented to the observer prior to the focal line contained a single line located $1.2 \mathrm{~cm}$ below a fixation point in the center of the viewing screen. The length of this contextual line was $1.4,1.9,4.2$, or 5.6 $\mathrm{cm}$. The test array contained the $2.8-\mathrm{cm}$ focal line as well as the contextual line that had been present prior to the test array. This contextual line was in the same spatial location as it had been prior to the presentation of the focal line; that is, it was centered $1.2 \mathrm{~cm}$ below and parallel to the focal line. Thus, on any given trial, the contextual stimulus was present both prior to and during judgment of the focal stimulus.

Focal judgments were obtained using the same graded-series comparison scale as in the previous experiment. Again, in order to en- 
courage observers to use a wide range of the response scale, two filler stimuli with focal lengths of 2.4 and $3.2 \mathrm{~cm}$ were included in the design. Responses to these filler stimuli were not included in the data analysis.

Finally, there were six practice stimuli which consisted of only a focal line. The focal lengths for these stimuli were $2.4,2.6,2.8$, $2.8,3.0$, and $3.2 \mathrm{~cm}$.

\section{Procedure}

The procedure for the present experiment was the same as that for Experiment 1 except as noted below. Each observer proceeded through a random order of the six practice trials, which was followed by a random order of the four test and two filler trials. For 18 of the 36 observers, a trial consisted of a 5-sec presentation of the adapting contextual line, during which the observers were instructed to fixate the small spot $1.2 \mathrm{~cm}$ above the line. This adaptation period was followed by the presentation of the test array containing both the focal and the contextual lines for up to $10 \mathrm{sec}$, during which the focal judgment was to be made. This was referred to as the " 5 -sec combined" condition. For the remaining 18 observers, trial consisted of a 60 -sec presentation of the adapting contextual line followed by the presentation of the focal and contextual lines for up to $10 \mathrm{sec}$. This was referred to as the " 60 -sec combined" condition. The interstimulus interval was $1 \mathrm{sec}$ for both conditions. Each trial was separated by a 20 -sec dark interval. After proceeding through the first random order of the four test and two filler trials, the observers proceeded through three more random orders of these six trials, with a 2-min break between the second and third random orders. In all, each of the 36 observers made 30 focal judgments-six practice judgments and four judgments of each of the four test and two filler stimuli. The procedure required approximately $18 \mathrm{~min}$ for observers in the 5 -sec condition and $45 \mathrm{~min}$ for observers in the $60-\mathrm{sec}$ condition.

\section{Results and Discussion}

The responses to the $2.8-\mathrm{cm}$ "no-context" focal line contained in the practice series were averaged to estimate the point of subjective equality (PSE) of the $2.8-\mathrm{cm}$ focal line for each group of observers. The mean of the 36 responses (two responses from each of 18 observers) was $2.57 \mathrm{~cm}$ for the 5 -sec combined conditions and 2.63 for the 18 observers in the 60 -sec combined condition. This consistent underestimation of objective focal length was similar to that reported in Experiment 1. Focal judgments were converted into deviations from PSE by subtracting PSE from the mean judged length of each test stimulus for both the 5- and 60-sec combined conditions. The results of combining simultaneous and sequential modes of presentation for 5 and $60 \mathrm{sec}$ of prior contextual exposure are shown in Figure 2.

The data for the 5-sec combined and the 60-sec combined conditions were analyzed separately in 4 (contextual lengths) $\times 4$ (replications) $\times 18$ (observers) withinsubject analyses of variance. For the 5-sec combined condition, the main effect of contextual length was not statistically significant $[F(3,51)<1]$, indicating that variations of contextual length did not have differential effects on focal judgments. However, the main effect of contextual length was statistically significant in the 60-sec combined condition $[F(3,51)=11.13, p<.01]$, which indicates that the variations in contextual lengths affected focal judgments. This effect (see the curve labeled "60 sec combined" in Figure 2) is in the direction of contrast of perceived focal length, although it should be noted that there

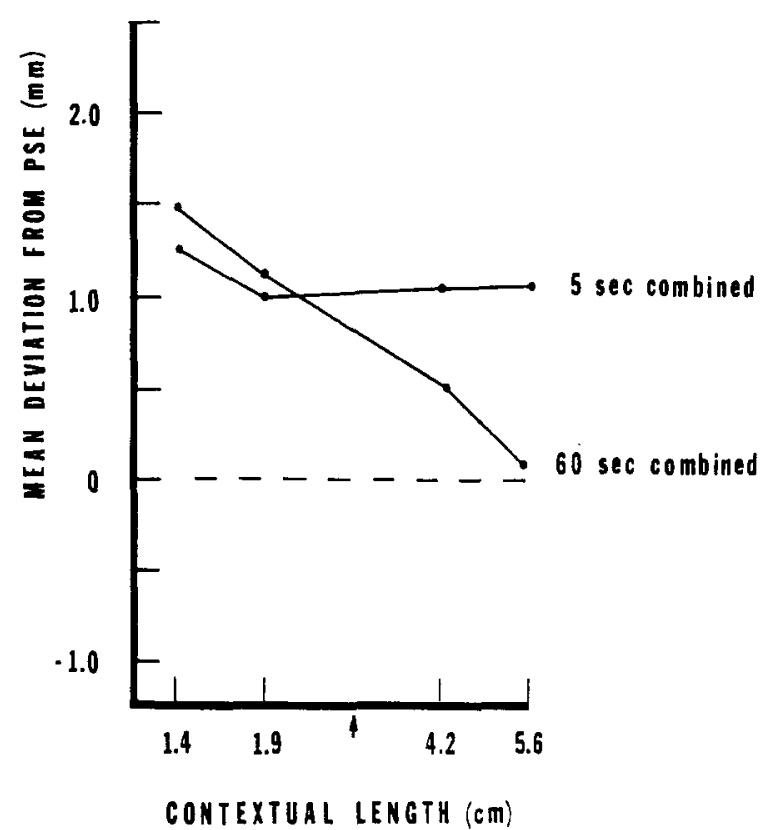

Figure 2. Mean deviations from PSE in millimeters for the 5-sec combined and 60-sec combined conditions of Experiment 2. The abscissa is a logarithmic scale of length. The arrow on the abscissa indicates the focal length $(2.8 \mathrm{~cm})$ for the test stimuli.

is no underestimation of focal length apparent in Figure 2. Thus, despite the presence of the contextual line simultaneously with the focal line, which generally results in length assimilation, the prior presentation of the contextual line resulted in length contrast. No other main effect or interaction reached statistical significance in either analysis.

Figure 3 shows that the length contrast resulting from the 60 -sec combined presentation of the contextual stimulus (Experiment 2) was markedly less than the contrast produced by the $60 \mathrm{sec}$ of contextual exposure in the aftereffect paradigm of Experiment 1. A 2 (conditions) $\times 4$ (contextual lengths) $\times 4$ (replications) $\times 18$ (observers) mixed analysis of variance evaluated the effects of the 60 sec aftereffect versus the 60-sec combined conditions of contextual exposure. Conditions was a between-subjects factor. The main effect of contextual line length was statistically significant $[F(3,102)=65.3, p<.01]$. Additionally, there was a significant conditions $\times$ contextual lengths interaction $[F(3,102)=10.12, p<.01]$, indicating that the slopes of the curves representing the 60 -sec aftereffect data (Experiment 1) and the 60-sec combined illusion and aftereffect data (Experiment 2) are significantly different. The direction of the slope difference indicates that the presence of the contextual line during the focal judgment in the 60-sec combined condition significantly reduced the magnitude of the contrast of perceived focal length relative to the baseline 60 -sec aftereffect data of Experiment 1.

In summary, the results of the present experiment again emphasize the importance of both mode of presentation and contextual exposure duration in determining length 


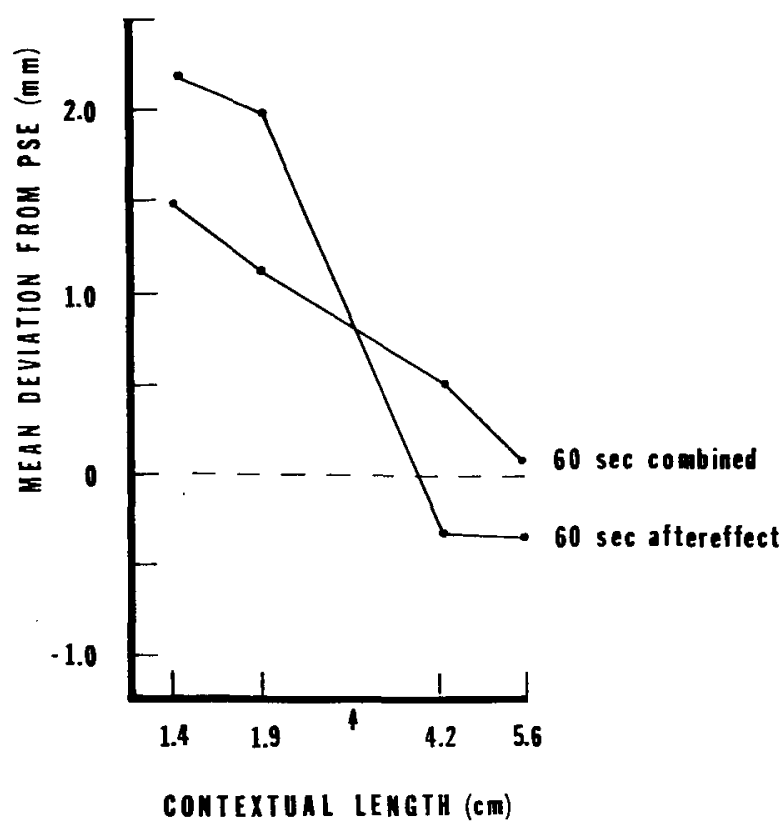

Figure 3. Mean deviations from PSE in millimeters for the 60sec aftereffect condition of Experiment 1 and the 60-sec combined condition of Experiment 2. The abscissa is a logarithmic scale of length. The arrow on the abscissa indicates the focal length $(2.8 \mathrm{~cm})$ for the test stimuli.

assimilation and length contrast. When prior and simultaneous contextual exposure durations were about the same, as in the 5-sec combined condition, there was no differential effect of variation of contextual length of focal judgments; there was no assimilation or contrast. It seemed as if the contrast produced by the 5-sec prior exposure to the contextual line was "cancelled" by the assimilation produced by the simultaneous presentation of the contextual and focal lines within a given trial. On the other hand, when there was an asymmetry in the duration of prior and simultaneous contextual exposure, as in the 60-sec combined condition, the pattern of focal judgments indicated length contrast. Thus, although variations of contextual exposure duration appear to affect the magnitude of observed length contrast independent of mode of presentation, the shift from length assimilation to length contrast seems to be mainly under the control of mode of presentation.

\section{GENERAL DISCUSSION}

The present research indicated that the mode of presentation of the contextual and focal stimuli determined whether assimilation or contrast of perceived length occurred in the parallel lines configuration. Additionally, the observed contrast in the sequential presentation condition increased as the duration of contextual exposure increased. Girgus and Coren (1982) have reported plasticity of contrast with variations of inspection time for the Delboeuf, Ebbinghaus, and Ponzo illusions.
The finding of assimilation of perceived focal length with simultaneous presentation and contrast with sequential presentation is consistent with Coren and Girgus's (1978) "pool and store"' model. As pointed out in the introduction, this model proposes that assimilation results when contextual and focal stimuli are close enough to be glimpsed simultaneously, and hence "averaged," based on "some normal judgmental processes involved in vision" (Girgus \& Coren, 1982). Conversely, when the contextual and focal stimuli are glimpsed sequentially, these same judgmental processes produce contrast. Thus, in the present Experiment 1, the simultaneous presentation of contextual and focal stimuli (the illusion condition) resulted in assimilation, but sequential stimulus presentation (the 5- and 60-sec aftereffect conditions) resulted in contrast.

It is important to note the difference in the magnitude of the length contrast produced by 5 and $60 \mathrm{sec}$ of prior contextual exposure in Experiment 1 (see Figure 1). Although the direction of length distortion (assimilation or contrast) seems to be determined by the mode of presentation of contextual and focal stimuli, the magnitude of the distortion is clearly influenced by contextual exposure duration. At present, the "pool and store" model does not directly address the issue of magnitude of distortion.

The effect of mode of presentation suggests that traditional neural fatigue explanations of contrast (e.g., Coltheart, 1971) may not apply to length contrast. If fatigue due to prolonged stimulation of length-sensitive neural units by the contextual line was responsible for length contrast, then it would seem that both the illusion and 5-sec aftereffect conditions should produce similar effects. Both effects would be assimilation if the hypothetical fatigue had not had enough time to build up, or both effects would be contrast if the fatigue had built up. However, the data from Experiment 1 show that $5 \mathrm{sec}$ of contextual exposure simultaneous with the focal line produced assimilation and $5 \mathrm{sec}$ of contextual exposure prior to the focal judgment produced contrast. The mode of presentation of contextual and focal stimuli seems more critical in the determination of assimilation or contrast than neural fatigue models would suggest. It could be argued that this comparison of the 5-sec illusion and aftereffect conditions is inappropriate, since there is the potential for fatigue of units sensitive to both the contextual and focal lines in the simultaneous condition as opposed to only the contextual line in the sequential condition. However, this would not be expected to affect the pattern of results, since a general observation is that a stimulus does not distort its own judgment (i.e., exposure to a $2.8-\mathrm{cm}$ contextual line does not distort the judgment of a subsequently viewed 2.8-cm focal line; Brigell \& Uhlarik, 1979).

The "pool and store" model suggests a general scheme for classifying distortions of linear extent as assimilation or contrast effects. According to Girgus and Coren (1982), "When the parts of the stimulus are spatially proximal, they are presumably sampled in a single glance, and hence 
are pooled or assimilated. On the other hand, when the parts of the stimulus are far away from each other spatially, they must be sampled in successive glances, with contrast between them the direct consequence"' (p. 559). The present research suggests that this general statement of the "pool and store" model be extended to include temporal proximity as an additional determinant of assimilation or contrast of perceived focal length. Temporal proximity (with a lower limit of simultaneity) of contextual and focal stimuli produces assimilation of linear extent, and temporal "distance" (sequential presentation) produces contrast. Thus, the "store" part of the "pool and store" model should not be thought of as applying only to what is typically referred to as the illusion paradigm; that is, spatial separation of contextual and focal stimuli is not a necessary condition for producing perceptual "storing." Rather, any experimental manipulation which encourages the observer to sample the contextual and focal stimuli in different or multiple glances should produce "storing," and hence contrast, according to the model. Put another way, either spatial or temporal separation should be capable of producing contrast. Coren and Girgus (1978) suggest this possibility in regard to the Delboeuf and Müller-Lyer figures.

Girgus and Coren (1982) suggest that one criterion for determining whether stimuli are distant enough to produce the "store" strategy resulting in contrast is whether the stimuli are subfoveal or suprafoveal. Presumably, stimuli that both project onto the fovea are proximal enough to be "pooled," resulting in assimilation; when only one part of the stimulus array projects onto the fovea, the array must be successively sampled, resulting in contrast. This statement of the model represents an important contribution in that it leads to directly testable predictions. For example, the contextual and focal stimuli in the illusion condition of Experiment 1 were spatially separated, yet assimilation of perceived focal length was observed. In that illusion condition, the spatial separation of the stimuli was $1.2 \mathrm{~cm}$ at a viewing distance of $77 \mathrm{~cm}$. This separation is less than $1^{\circ}$ of visual angle, and the finding of assimilation under these conditions is consistent with the use of the subfoveal-suprafoveal criterion of spatial separation. It would therefore be expected that if the contextual and focal stimuli in the parallel-lines configuration were presented simultaneously, but with a greater spatial separation (e.g., greater than $3^{\circ}$ of visual angle), a shift from assimilation to contrast should be observed. However, Schiano and Girgus (1981) reported assimilation in the parallel-lines configuration with more than a $4^{\circ}$ separation of the contextual and focal stimuli. The failure to produce length contrast under these conditions of suprafoveal presentation calls the subfoveal-suprafoveal criterion of spatial separation into question. Additionally, Jordan and Uhlarik (1984) reported that a vertical spatial separation of the contextual "fins" from the focal "shaft" in a horizontally oriented Müller-Lyer figure resulted in length contrast. However, the spatial separation was less than $3^{\circ}$. This finding is also inconsistent with the subfoveal-suprafoveal criterion, which would have predicted length assimilation under Jordan and Uhlarik's conditions. Possibly, the spatial separation of the contextual and focal lines relative to the size of the contextual and focal lines is more important than their absolute separation in visual angle in determining whether the perceptual system "pools" or "stores," resulting in either assimilation or contrast.

In summary, the "pool and store" model may serve as a good description of the shift from assimilation to contrast in the parallel-lines configuration. Additionally, the model seems to generalize well to other distortions of linear extent and area, such as the Delboeuf, Ebbinghaus, and Ponzo illusions (Girgus \& Coren, 1982) and the Müller-Lyer figure (Jordan \& Uhlarik, 1984). Finally, Jordan and Uhlarik (1983) also reported length contrast when the focal "shaft" of the Müller-Lyer figure was judged subsequent to either 5 or $60 \mathrm{sec}$ of exposure to the contextual "fins." This Müller-Lyer "aftereffect" is also compatible with the "pool and store" model and is very similar to the aftereffect of parallel lines. It would be interesting to analyze other distortions of linear extent, such as the Baldwin figure, for their compatibility with the model. It may be that many configurations that have traditionally been thought of as producing only assimilation or only contrast will actually produce both effects in ways predictable from Coren and Girgus's model, as has been found for the parallel-lines and Müller-Lyer configurations.

The present reserch suggests that temporal, as well as spatial, proximity is an important determinant of the shift from assimilation to contrast. Of course, it might simply be that when two stimiuli (e.g., parallel lines) are spatially distal, temporal separation of the stimuli is inevitable due to successive sampling; that is, spatial separation may be redundant with temporal separation. Additionally, the "pool and store" model will need to accommodate the finding of contrast with subfoveal separation of contextual and focal elements of the Müller-Lyer figure (Jordan \& Uhlarik, 1984) and assimilation with suprafoveal separation of contextual and focal lines in the parallel lines figure (Schiano \& Girgus, 1981). These data indicate that the amount of spatial separation needed to produce the shift from assimilation to contrast is not related to the fovea and may depend more on the relations among the stimulus elements in the array.

\section{REFERENCES}

Blakemore, C., Carpenter, R. H. S., \& Georgeson, M. A. (1970). Lateral inhibition between orientation detectors in the human visual system. Nature, 228, 37-39.

Blakemore, C., Nachmias, J., \& Sutton, P. (1970). The perceived spatial frequency shift: Evidence for frequency-selective neurons in the human brain. Joumal of Psychology (London), 210, 727-750.

BRIGELL, M., \& UHLARIK, J. (1979). The relational determination of length illusions and length aftereffects. Perception, 8, 187-197.

COLTHEART, M. (1971). Visual feature-analyzers and aftereffects of tilt and curvature. Psychological Review, 78, 114-121.

Coren, S., GiRgUS, J. S. (1972). A comparison of five methods of 
illusion measurement. Behavior Research Methods \& Instrumentation, 4, 240-244.

COREN, S., \& GIRGUS, J. S. (1978). Seeing is deceiving: The psychology of visual illusions. Hillsdale, NJ: Erlbaum.

GiBSON, J. J., \& RADNER, M. (1937). Adaptation, aftereffect, and contrast in the perception of tilted lines: I. Quantitative studies. Journal of Experimental Psychology, 20, 453-467.

Girgus, J. S., \& COREN, S. (1982). Assimilation and contrast illusions: Differences in plasticity. Perception \& Psychophysics, 32, 555-561.

JASTROW, J. (1892). On the judgments of angles and positions of lines. American Journal of Psychology, 5, 214-221.

JORDAN, K. (1982). Assimilation and contrast of perceived length: Tests of models of neural interaction. Unpublished doctoral dissertation, Kansas State University.

JORDAN, K., \& UHLARIK, J. (1983, November). The Mueller-Lyer aftereffect: Temporal separation produces length contrast. Paper presented at the meeting of the Psychonomic Society, San Diego.
JoRDAN, K., \& UhLARIK, J. (1984, November). Spatial separation produces length contrast in the Mueller-Lyer figure. Paper presented at the meeting of the Psychonomic Society, San Antonio.

Klein, S., Stromeyer, C. F., \& Ganz, L. (1974). The simultaneous spatial frequency shift: A dissociation between the detection and perception of gratings. Vision Research, 14, 1421-1432.

MaGNusSEN, S., \& KuRTENBACH, W. (1980). Linear summation of the tilt illusion and tilt aftereffect. Vision Research, 20, 39-42.

Schiano, D. J., \& Girgus, J. S. (1981). A second look at the parallel line illusion. Paper presented at the meeting of the Eastern Psychological Association, New York.

(Manuscript received October 22, 1984; revision accepted for publication March 14, 1985.) 